\title{
A non-enzymatic Electrochemical Sensor Based on rGO-PPy for Rapid and Sensitive Determination of Histamine in Meat
}

\author{
Bobo Hu ${ }^{1}$, Nana Zhang ${ }^{2}$, Huihui $\mathrm{Li}^{1,{ }^{*},}$, Chong Sun ${ }^{2,3, *}$ \\ ${ }^{1}$ Jiangsu Collaborative Innovation Centre of Biomedical Functional Materials, Jiangsu Key Laboratory \\ of Biomedical Materials, School of Chemistry and Materials Science, Nanjing Normal University, \\ Nanjing 210023, PR China \\ ${ }^{2}$ Institute of Agricultural Products Processing, Jiangsu Academy of Agricultural Sciences, Nanjing \\ 210014, PR China \\ ${ }^{3}$ Jiangsu Key Laboratory for Food Quality and Safety-State Key Laboratory Cultivation Base of \\ Ministry of Science and Technology, Nanjing 210014, PR China \\ "E-mail: huihuili@njnu.edu.cn (H.H. Li); sunchong@jaas.ac.cn (C. Sun)
}

doi: $10.20964 / 2021.05 .06$

Received: 10 December 2020 / Accepted: 8 February 2021 / Published: 31 March 2021

Sensitive and reliable quantification of histamine (His) is essential to ensure food safety. In this study, a sensing material consisting of reduced graphene oxide/polypyrrole (rGO-PPy) composites is successfully prepared by a two-step synthesis method and verified by transmission electron microscopy (TEM), Fourier transform infrared (FTIR) spectroscopy, ultraviolet-visible (UV-vis) spectroscopy, Xray diffraction (XRD) and X-ray photoelectron spectroscopy (XPS). Based on rGO-PPy, an electrochemical method is established, and the electrochemical behaviours are studied by cyclic voltammetry (CV), electrochemical impedance spectroscopy (EIS) and differential pulse voltammetry (DPV). The experimental parameters, such as the detection method, the concentration and volume of rGO-PPy modified on GCE, and the $\mathrm{pH}$ value of the buffer solution, are optimized. Under the optimized conditions, the linear equation of the electrochemical sensor to His is $I(\mu \mathrm{A})=0.01047 \mathrm{c}+0.1842\left(\mathrm{R}^{2}=\right.$ 0.9970 ) with a linear range of $10 \sim 800 \mu \mathrm{M}$, and the detection limit is $3.01 \mu \mathrm{M}$. Practical samples are also detected, which proves that the sensor has great application prospects. Therefore, this idea and method will make an excellent contribution to food safety.

Keywords: rGO-PPy; Histamine; Electrochemical sensor; Food safety

\section{FULL TEXT}


(C) 2021 The Authors. Published by ESG (www.electrochemsci.org). This article is an open access article distributed under the terms and conditions of the Creative Commons Attribution license (http://creativecommons.org/licenses/by/4.0/). 